\title{
P71 The Interrelationship Between the Alteration of Arterial Markers and Left Ventricular Diastolic Dysfunction in Metabolic Syndrome Subjects
}

\author{
Svetlana Solovjova*, Ligita Ryliskyte, Jelena Celutkiene, Jolita Badariene, Aleksandras Laucevicius
}

Vilnius University Hospital Santaros Clinics, Vilnius, Lithuania

\section{ABSTRACT}

We aimed to determine how the alteration of arterial stiffness (AS) affects the dynamics of left ventricular (LV) diastolic dysfunction (DD) in metabolic syndrome (MetS) subjects during longitudinal observation (3.8 \pm 0.6 years).

Methods: A follow-up study was carried out in 573 (53.4 \pm 5.8 years, 211 men [36.8\%]) MetS subjects without overt atherosclerotic disease. Carotid-to-femoral pulse wave velocity (cfPWV), augmentation index (AIxHR75), mean aortic pressure (mAP), central pulse pressure(cPP) were assessed by applanation tonometry. Diastolic function (LVDF) was defined according to the 2016ESC Guidelines for diagnosis of acute and chronic heart failure.

Results: Depending on the LVDD dynamics analysis focused on the comparison of arterial markers in 2 subgroups (Figure 1): GRI (LVDD improved: $22 \%, n=126$ ) and GRIII (LVDF worsened: 19.2\%, $n=110$ ). After 3 years in both groups (all $p<0.05$ ): decreased HR (GRI $66.27 \pm 10.09$ to $62.15 \pm 8.86 \mathrm{~b} / \mathrm{min}$; GRIII $65.17 \pm 9.41$ to $62.07 \pm 8.51 \mathrm{~b} / \mathrm{min}$ ) and $\mathrm{mAP}$ (GRI $107.60 \pm 10.89$ to $100.48 \pm 16.55 \mathrm{mmHg}$; GRIII $104.07 \pm 10.94$ to $99.73 \pm 13.17 \mathrm{mmHg}$ ). cfPWV and AIx significantly increased only in GRIII (cfPWV: $8.25 \pm 1.36$ to $8.68 \pm 1.73 \mathrm{~m} / \mathrm{s}$; AIx $23.16 \pm 9.94$ to $25.16 \pm 10.08 \%$ ). These results confirm, that the negative dynamics of LVDF is associated with the progression of the arterial stiffening. For more accurate evaluation of the relationship between alterations of AS indices and the dynamics of LVDD the conditional random forests analysis was performed. Random forests analysis demonstrated that cfPWV, BMI, weight had prognostic significance for the worsening of LVDF.

Conclusion: In conclusion, the increase of cfPWV and AIx are significantly associated with negative dynamics of LVDF. In MetS subjects progression in arterial stiffening, assessed as an increase in cfPWV, significantly predicts the worsening of the LVDF. Our findings suggest that increased arterial stiffness is a pathogenetic factor of LVDD.

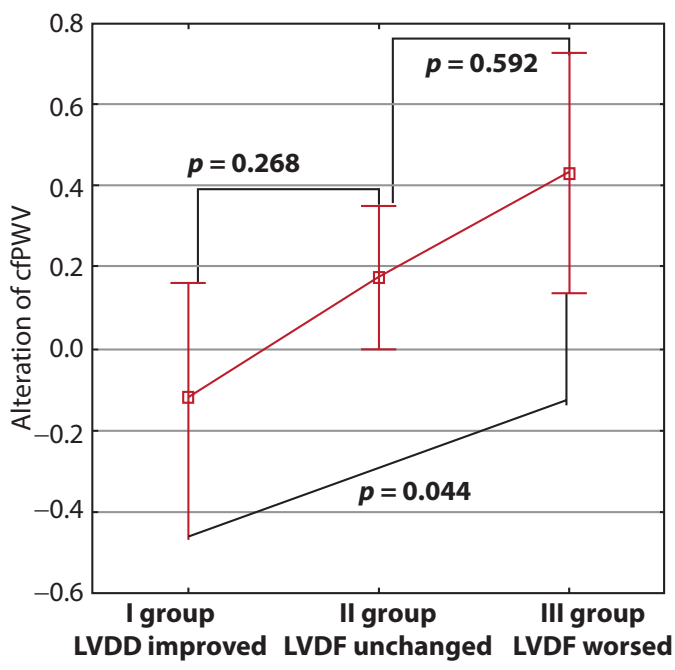

Figure 1

(C) 2019 Association for Research into Arterial Structure and Physiology. Publishing services by Atlantis Press International B.V. This is an open access article distributed under the CC BY-NC 4.0 license (http://creativecommons.org/licenses/by-nc/4.0/). 